\title{
Caracterización de la educación superior a distancia, en las universidades colombianas
}

Olga Lilihet Matallana Kuan ${ }^{1}$

Marleny Torres Zamudio ${ }^{2}$

Resumen

El estudio de caracterización de la educación superior a distancia en las universidades Colombianas presenta la situacion actual de este tipo de educacion, pues aunque hay estudios estadisticos que contemplan la educacion superior, pocos son los que se detienen en la educacion distancia, considerando que esta representa el $10 \%$ del total de educación superior en el país. La principal fuente de información es el análisis y exploracion de los datos suministrados por el Sistema Nacional de Información de la Educación Superior (SNIES) y el O bservatorio Laboral para la Educación, sistemas oficiales de información del Ministerio de Educación Nacional (M EN) que brindan amplia información estadística sobre la educación superior del país.

Palabras elave: Educación a Distancia, universidades, estudiantes matriculados, programas, graduados. 
Olga Lilihet M atallana Kuan y M arleny Torres Zamudio

Caracterización de la educación superior a distancia, en las universidades colombianas, artículo

producto de la investigación

\title{
A characterization of distance learning higher education in Colombian universities
}

\begin{abstract}
A.bstract
This study of characterization of higher and distance education in Colombian universities presents the current status of distance education. While we have statistical studies on higher education, they are not specific on distance education, notwithstanding the fact that it represents $10 \%$ of higher education in our country. The main source of information is the analysis and exploration of the data provided by the National Higher Education Information (SNIES) and the Labor Observatory for Education, official information systems from the Ministry of Education, which provide extensive statistical information about higher education in Colombia.
\end{abstract}

Key words: Distance education, universities, registered student, programs, graduates.

Recibido: 14 Diciembre de 2010

Aceptado: 24 Febrero de 2011

\section{Introducción}

La Educación Superior a distancia en Colombia se presenta como una alternativa a la educación tradicional, y se define como el "conjunto de estrategias metodologicas y tecnologicas para establecer la comunicacion entre quienes participan en un mismo proceso educativo aunque no coincidan en el tiempo 0 lugar de estudio" (M oreno, 2001). Dadas las potencialidades educativas que brinda, esta metodologia adquiere cada vez mas relevancia pues cambia los paradigmas educativos y orienta las instituciones de educacion superior a ser centros generadores de conocimiento, no solo porque se superan barreras de espacio y tiempo sino porque no ásla al estudiante y. le permite seguir, su rol laboral, familiar o social, generando importantes posibilidades estrategicas de desarrollo mediante la virtualidad.

La Resolución 2755 de 2006, emanada por el MEN, delimita la educación superior a distancia como "aquell la metodología educativa que se caracteriza por util izar ambientes de aprendizaje en los cual es se hace uso intensivo de diversos medios de informacion y comunicacion y de mediaciones pedagogicas que 


\section{Revista de}

permiten crear una dinámica de interacción orientada al aprendizaje autónomo y abierto, superar la docencia por exposicion y el aprendizaje por recepción, así como las barreras espacio-temporales y las limitaciones de la realidad objetiva mediante simulaciones virtuales, adelantar relaciones reales o mediadas y facil itar aprendizajes por indagacion y mediante la colaboracion de diversos agentes educativos" 3 . Pero icual, es el estado de la educación superior que se ofrece bajo esta metodología en el país?

\section{Metodología}

La investigación se orienta hacia la determinación de las características de la educacion superior a distancia en Colombia mediante la seleccion de las universidades partiendo de los parámetros brindados por el SNIES y el Observatorio Laboral para la Educacion. Una vez identificadas las universidades, se procede a real izar la recoleccion de la informacion pertinnente a la investigacion mediante una guia de observación (anexo 1) que orienta el proceso como instrumento de recoleccion de informacion.

Las dimensitones consideradas en el estudio fueron : universidades, definidas como las Instituciones de Educacion Superior del pals, de origen publico o privado: programas, que hace referencia al número de programas ofrecidos, las areas, el nivel de formacion y programas activos, inactivos y acreditados; estudiantes matriculados, dimensión que responde al porcentaje de cobertura educativa de la metodologia a distancia en el pals respecto al total nacional; graduados, que recoge la informacion que se refiere a los graduados, considerando las areas académicas y los departamentos del pals que presentaron un mayor numero de graduados en metodologia a distancia' Ingreso base de cotizacion, IBC, que corresponde al indice de graduados en 2008 (ultimo ano registrado en el aplicativo consultado) que a la fecha de la consulta habían obtenido trabajo y al salario alcanzado en promedio segun el nivel de formacion.

\section{Resultados}

Educación superior a distancia en América Latina

La A sociación Colombiana de Univerșidades, A scun, en el documento "Políticas y estrategias para la Educacion Superior en Colombia, 2006-2010" (2007), dice que ha habido, un fuerte crecimiento en la demanda de educacion superior en el mundo, en razón de los cambios demográficos. "En A frica, A sia y A mérica Latina,

3 Resolución 2755 de 2006, emanada del Ministerio de Educación Nacional de Colombia. 
Olga Lilihet M atallana Kuan y M arleny Torres Zamudio

Caracterización de la educación superior a distancia, en las universidades colombianas, artículo

producto de la investigación

el fuerte crecimiento demográfico ha contribuido a aumentar la afluencia a los niveles de primaria y secuncaria, provocando aś un aumento de los estudiantes en la enseñanza superior, aunque en proporciones menores que en Europa o A merica del Norte". En America Latina se vive la tercera reforma de la educacion superior, caracterizada por masificacion, regulaciones e internacional|zacion de esta educacion; se avanza en temas como la diversidad, la inclusion y el desarrollo institucional. (Instituto Internacional de la Unesco para la Educación Superior en América Latina y el Caribe, IESALC, Unesco, 2006) y se resalta una tendencia de dinamismo de la educacion superior, que puede sostenerse a largo plazo. Según Misas (2004), las universidades virtuales y la educaciọn a distancia asumen cada vez más posibilidades de aportar soluciones en terminos de cobertura representando una importante oportunidad para que modelos de educacion diferentes al tradicional cobren mas relevancia en el ambito educativo.

La Unesco, en el Informe sobre la educación superior en América Latina y el Caribe 2000-2005, plantea una radiografia de la educación superior a distancia en Latinoamerica, desglosada asi::

México cuenta con más de cincuenta años de experiencia en educación a distancia en diversos niveles educativos, con instituciones como la Universidad Nacional Autonoma de México, Unam, la Universidad Pedagogica Nacional, UPN y la Red Nacional de EducaciónA biertay a Distancia. Panama esta en una etapa de adaptación y formacion de las destrezas tecnologicas; no obstante, al gunas universidades ofrecen programas de educación a distancia. Guatemala cuenta con algunos ejemplos de educación a distancia como la Universidad Rafael Landivar, URL.

Son quince las universidades latinoamericanas que ofrecen programas exclusivamente en la modalidad a distancia. Respecto a programas a nivel de prearado, en 2005 solo se ofrecian 23 programas a traves de seis universidades en Colombia, cuatro en M éxico y una en Bolivia. La oferta es de al menos una carrera por cada área disciplinar, predominando en los programas de ciencias humanas (40\%), ciencias sociales (26\%) y ciencias sagradas (13\%). En el nivel de postarado se of recían treinta programas distribuidos en nueve instituciones en las que dominan las areas de ciencias humanas $(47 \%)$ y ciencias de la salud $(20 \%)$.

En lo que respecta a cobertura, Colombia en 2005 "mostró una cobertura inferior a la que presentaron en 2003 varios de los paises latinoamericanos con desarrollo simil ar como A rgentina que llego aproximadamente al $60 \%$, Panama que alcanzo el $51 \%$, Chile un $47 \%$, Costa Rica (44\%) Venezuela (43\%), Uruguay (41\%), Bolivia (38\%), República Dominicana $37 \%$ ), Peru (34\%), Cuba $(34 \%) . " 5$ Colombia obtuvo una cobertura de $24.6 \%$. Es evidente que estas

4 Unesco, "Hacia las Sociedades del Conocimiento. Informe Mundial”, 2005, p. 97

5 Zarur, Xiomara; Rodriguez, Juan Carlos: “Estudio Nacional sobre la Evolución, Situación Actual y Perspectivas de la Educación Superior en Colombia 2002-2006". Estudio elaborado para el lesalc-Unesco, septiembre 2006, pág. 46. 
Revista de investigaciones UNAD

Volumen 10. Número 1. Junio 2011

tendencias han cambiado las características y la concepción de la educación a distancia, pues "L as profundas transformaciones ocurridas en las universidades latinoamericanas, expresadas en un proceso de masificación, feminizacion, privatizacion, regionalizacion, diferenciacion y segmentacion, sumado a los propios cambios, de esas sociedades sumidas en un proceso de urbanizacion, de cambio demografico, de transtormacion productiva y de apertura economica, cambiaron sustancialmente el rol y las caracteristicas de los estudiantes". . Esta tendencia es una oportunidad para la educacion a distancia y genera un mayor compromiso de las instituciones ante los retos que se imponen.

\section{La educación superior a distancia en Colombia}

En el país, según datos del Estudio Nacional sobre la Evolución Situación Actual y Perspectivas de la Educación Superior en Colombia 2002-2006, la tasa de cobertura bruta en el periodo 2002-2005 se incremento de 20.8\% a $24.6 \%$ y para2008 es de 35.5\%, mostrando un fuerte crecimiento en cobertura debido al incremento del sector privado que representa el $51,5 \%$ del total en 2005. La concentracion de la oferta de educacion superior sigue en cinco ciudades:' Bogota con el 33.1\% del total nacional, Antioquia con el 13.5\%, Valle con $8.3 \%$, Atlántico con $6.3 \%$ y Santander con $6.0 \%$. Las áreas con mayor matrícula son Ingenieria, Arquitectura y Urbanismo, seguidas de Economía, Administración y Contaduría. La tasa de deserción que se presentaba en 2004 era de $47 \%$ y en 2008, de 44.9\%, porcentaje bastante elevado que exige implementar urgentemente estrategias de retención estudiantil.

De acuerdo con González(1992), Briceño (1991), Parra y Jaramillo(1985) y O Orozco et al, las tendencias tranșformadoras que se han producido en los últimos años en la educación superior colombiana son: acelerada expansion del tercer nivel educativo en cuanto a matricula, prol iferacion de instituciones, programas de estudjo y planta de profesores, diversificacion de modal idades y niveles de educacion con el consecuente desarrollo curricular, ampliacion de ofertas educativas con la creacion de la educacion abierta y a distancia, creciente privatizacion y feminizacion, contraccion del mercado laboral y devaluacion de los estudios profesionales, incipiente desarrollo de la investigación y de los estudios de posgrado e insuficiencia e inequidad en la distribucion de los subsidios por parte del Estado.

\section{Estructura de la Educación Superior en Colombia}

La educación superior en el país es regulada por la Ley 30 de 1992, que es la que define varios tipos de instituciones según su natural eza y objetivos (Instituciones Técnicas Profesionales, Instituciones Tecnologicas, Instituciones

6 lesalc-Unesco: "Informe sobre la Educación Superior en América Latina y el Caribe 2000-2005. La metamorfosis de la educación superior, 2006, pág. 15. 
Olga Lilihet M atallana Kuan y M arleny Torres Zamudio

Caracterización de la educación superior a distancia, en las universidades colombianas, artículo producto de la investigación

Universitarias y Universidades). Según el Consejo Nacional de Acreditación, las universidades son "I as instituciones que acrediten su desempeño con criterio de universalidad en las siguientes actividades: la investigacion cientifica o tecnologica, la formacion academica en profesiones o disciplinas y la produccion, desarrollo y transmision del conocimiento y de la cultura universal y nacional, Estas instituciones están igualmente facultadas para adelantar programas de formacion en ocupaciones, profesiones o disciplinas, programas de especialización, maestrias, doctorados y post-doctorados, de conformidad conla Ley".

Deacuerdo con el M EN los programas de pregrado preparan para el desempeño de ocupaciones o para el ejercicio de una profesion o disciplina determinada, de naturaleza tecnologica o cientifica; los programas de postgrado denominados especializaciones posibilitan el perfeccionamiento en la misma ocupación, profesion, disciplina 0 áreas afines o complementarias. L os demas postgrados, como maestrias, doctorados y post doctorados, basan su quehacer en la investigacion como fundamento y ambito necesario de su actividad.

Las universidades se clasifican en oficiales y privadas; las instituciones oficiales o públicas son del orden nacional, departamental o municipal (M ontenegro, 1994). Las universidades privadas, pueden clasificarse como Corporacion o Fundación. En lo que respecta a áreas académicas, todos los programas de igual o distinta modalidad educativa se ubican en una de las nueve areas del conocimiento; 1. A gronomía, Veterinaria y afines; 2. B ellas A rtes;' 3. Ciencias de la Educación; 4. Ciencias de la Salud; 5. Ciencias Sociales, Derecho y Ciencias Politicas;' 6. Economia, Administracion, Contaduría y afines; 7 . Humanidades y Ciencias Religiosas; 8 . Ingeniería, A rquitectura, U rbanismo y afines y 9. Matemáticas y Ciencias Naturales.

\section{Universidades}

Según los datos del SNIES, de las 282 instituciones de educación superior, hay 79 registradas como universidades y las demas corresponden a Instituciones Técnicas Profesionales, Tecnologicas e Inșitituciones U niversitarias. Solo 55 univerșidades ofrecen programas de educacion a distancia, de las cuales treinta son universidades privadas y 25 universidades oficiales.

Programas

a) Número de programas ofrecidos a distancia respecto del número total de programas existentes a nivel nacional.

7 Para mayor información sobre la educación superior en Colombia, consúltese la página electrónica del Consejo nacional de acreditación, http://www.cna.gov.co. 
Revista de

investigaciones UNAD

Volumen 10. Número 1. Junio 2011

Tabla 1. Programas a distancia

\begin{tabular}{|c|c|c|c|}
\hline Año 2009 & Pregrado & Postgrado & Total \\
\hline Presencial & 4208 & 5678 & 9886 \\
\hline A distancia & 499 & 177 & 676 \\
\hline Total & 4707 & 5855 & 10562 \\
\hline \% respecto al total & 10,6 & 3,0 & 6,4 \\
\hline
\end{tabular}

Fuente: SNIES, Número de programas ofrecidos a distancia respecto del número total de programas existentes a nivel nacional

La oferta educativa de programas bajo la modalidad a distancia solamente representa en pregrado el $10.6 \%$ de la totalidad de programas, y a nivel de postgrado significa el $3 \%$ de ellos (Ver tabla1).

b) Número de programas académicos por nivel de formación y origen institucional.

Según los datos del SNIES para 2009, existen 676 programas a distancia en el pals, de los cuales un $53.8 \%$ es ofrecido por universidades oficiales y $46.15 \%$ por universidades privadas.

De acuerdo con el nivel de formación, un $5 \%$ de los programas que se ofrecen corresponde a carreras de tecnico profesional, un 25\% a carreras tecnológicas, un $43.7 \%$ a estudios universitarios, un $25 \%$ a especialización y solo un $1.18 \%$ corresponde a estudios de maestria. Notese que no existen en el pais doctorados ofrecidos bajo la modalidad a distancia. Las instituciones privadas ofrecen mas programas de postgrado, mientras que las universidades oficiales lo hacen más en estudios de pregrado (Ver tabla 2).

Tabla 2. Programas por nivel de formación

\begin{tabular}{|c|c|c|c|c|c|c|}
\hline & \multicolumn{3}{|c|}{ Pregrado } & \multicolumn{2}{|c|}{ Postgrado } & \\
\hline & Tecnológica & $\begin{array}{c}\text { Técnica } \\
\text { profesional }\end{array}$ & Universitaria & Especialización & Maestría & \\
\hline Oficial & 135 & 23 & 161 & 44 & 1 & 364 \\
\hline Privada & 34 & 11 & 135 & 125 & 7 & 312 \\
\hline Total & 169 & 34 & 296 & 169 & 8 & 676 \\
\hline
\end{tabular}

Fuente: SNIES. Datos por área académica 
Olga Lilihet M atallana Kuan y M arleny Torres Zamudio Caracterización de la educación superior a distancia, en las universidades colombianas, artículo producto de la investigación

c) Número de programas ofrecidos por nivel de formación en universidades oficiales.

De las 25 universidades oficiales que ofrecen programas a distancia, solo la UNAD se dedica exç lusivamente a esta modalidad con 49 programas en pregrado. Las otras universidades oficiales ofrecen programas a distancia y presenciales, En la modalidad a distancia se destacan: Ta Universidad del Quindio con 31 programas de pregrado, la Universidad del Tolima con 28 y la de Cartagena con 27. En lo que respecta a postarado, la UNAD tiene doce programas y la Universidad del Valle y la del Tolima, once cada una. En el nivel de Ma aestría, la U niversidad del Valle tiene un programa. Los programas de educacion a distançia en univerșidades oficiales se concentran en el nivel de formacion universitaria y tecnologica.

d) Número de programas ofrecidos por nivel de formación en universidades privadás.

De las treinta universidades privadas que brindan programas a distancia, la Universidad A ntonio Nariño es la que cuenta con una mayor oferta con 37 programas en pregrado. Le siguen la Universidad Santo Tomas con 34 programas, la Universidad A utónoma de Bucaramanga, UNA B, y la Católica de Oriente, cada una con 18 programas, y la Universidad Central y la de $M$ anizales con un programa bajo esta modali dad cada una. En lo que respecta a postgrado, siguen siendo la U, niversidad A ntonio N ariño y la Universidad Santo Tomas las que ofrecen más programas, 27 y 20 respectivamente. La Universidad El B osque brinda quince programas y la Escuela de A dministración de Negocios, Ean, once programas. A nivel de maestrías, la UNAB tiene cinco programas y, la de $M$ anizales uno, llegando a la concl usion de que la oferta de maestrias está en la universidad privada.

e) Número de programas por área académica y origen de la institución.

Tabla 3. Programas por área académica

\begin{tabular}{|c|c|c|c|c|c|c|c|c|c|c|c|c|}
\hline \multirow{3}{*}{$\begin{array}{c}\text { Año } \\
2009 \\
\text { Área }\end{array}$} & \multicolumn{6}{|c|}{ Pregrado } & \multicolumn{4}{|c|}{ Postgrado } & \multicolumn{2}{|c|}{ Total } \\
\hline & \multicolumn{2}{|c|}{$\begin{array}{l}\text { Tecnoló- } \\
\text { gica }\end{array}$} & \multicolumn{2}{|c|}{$\begin{array}{c}\text { Técnica } \\
\text { profesional }\end{array}$} & \multicolumn{2}{|c|}{$\begin{array}{l}\text { Universi- } \\
\text { taria }\end{array}$} & \multicolumn{2}{|c|}{$\begin{array}{l}\text { Especiali- } \\
\text { zación }\end{array}$} & \multicolumn{2}{|c|}{ Maestría } & & \\
\hline & $\begin{array}{l}\text { Ofi- } \\
\text { cial }\end{array}$ & $\begin{array}{l}\text { Pri- } \\
\text { vada }\end{array}$ & $\begin{array}{l}\text { Ofi- } \\
\text { cial }\end{array}$ & $\begin{array}{l}\text { Priva- } \\
\text { da }\end{array}$ & $\begin{array}{l}\text { Ofi- } \\
\text { cial }\end{array}$ & $\begin{array}{l}\text { Priva- } \\
\text { da }\end{array}$ & $\begin{array}{l}\text { Ofi- } \\
\text { cial }\end{array}$ & $\begin{array}{l}\text { Pri- } \\
\text { vada }\end{array}$ & $\begin{array}{l}\text { Ofi- } \\
\text { cial }\end{array}$ & $\begin{array}{l}\text { Pri- } \\
\text { vada }\end{array}$ & & \\
\hline $\begin{array}{l}\text { Agrono- } \\
\text { mía, vete- } \\
\text { rinaria } \\
\text { y afines }\end{array}$ & 25 & 4 & 4 & 0 & 4 & 0 & 2 & 4 & 0 & 0 & 35 & 8 \\
\hline
\end{tabular}




\section{Revista de}

investigaciones UNAD

Volumen 10. Número 1. Junio 2011

\begin{tabular}{|c|c|c|c|c|c|c|c|c|c|c|c|c|}
\hline \multirow{3}{*}{\begin{tabular}{|c|} 
Año \\
2009 \\
Área \\
\end{tabular}} & \multicolumn{6}{|c|}{ Pregrado } & \multicolumn{4}{|c|}{ Postgrado } & \multicolumn{2}{|c|}{ Total } \\
\hline & \multicolumn{2}{|c|}{$\begin{array}{l}\text { Tecnoló- } \\
\text { gica }\end{array}$} & \multicolumn{2}{|c|}{$\begin{array}{c}\text { Técnica } \\
\text { profesional }\end{array}$} & \multicolumn{2}{|c|}{$\begin{array}{l}\text { Universi- } \\
\text { taria }\end{array}$} & \multicolumn{2}{|c|}{$\begin{array}{l}\text { Especiali- } \\
\text { zación }\end{array}$} & \multicolumn{2}{|c|}{ Maestría } & & \\
\hline & $\begin{array}{l}\text { Ofi- } \\
\text { cial }\end{array}$ & $\begin{array}{l}\text { Pri- } \\
\text { vada }\end{array}$ & $\begin{array}{l}\text { Ofi- } \\
\text { cial }\end{array}$ & $\begin{array}{l}\text { Priva- } \\
\text { da }\end{array}$ & $\begin{array}{l}\text { Ofi- } \\
\text { cial }\end{array}$ & $\begin{array}{l}\text { Priva- } \\
\text { da }\end{array}$ & $\begin{array}{l}\text { Ofi- } \\
\text { cial }\end{array}$ & $\begin{array}{l}\text { Pri- } \\
\text { vada }\end{array}$ & $\begin{array}{l}\text { Ofi- } \\
\text { cial }\end{array}$ & $\begin{array}{l}\text { Pri- } \\
\text { vada }\end{array}$ & & \\
\hline $\begin{array}{l}\text { Bellas } \\
\text { artes }\end{array}$ & 0 & 1 & 0 & 0 & 1 & 0 & 0 & 0 & 0 & 0 & 1 & 1 \\
\hline $\begin{array}{l}\text { Ciencias } \\
\text { de la } \\
\text { Educa- } \\
\text { ción }\end{array}$ & 0 & 0 & 0 & 0 & 71 & 84 & 12 & 58 & 0 & 2 & 83 & 144 \\
\hline $\begin{array}{l}\text { Ciencias } \\
\text { de la } \\
\text { Salud }\end{array}$ & 8 & 2 & 0 & 0 & 9 & 0 & 2 & 0 & 1 & 0 & 20 & 2 \\
\hline $\begin{array}{l}\text { Ciencias } \\
\text { sociales y } \\
\text { humanas }\end{array}$ & 3 & 2 & 0 & 0 & 7 & 6 & 1 & 11 & 0 & 0 & 11 & 19 \\
\hline $\begin{array}{l}\text { Eco- } \\
\text { nomía, } \\
\text { Adminis- } \\
\text { tración, } \\
\text { Conta- } \\
\text { duría y } \\
\text { afines }\end{array}$ & 53 & 13 & 18 & 5 & 35 & 37 & 16 & 40 & 0 & 2 & 122 & 97 \\
\hline $\begin{array}{l}\text { Ingenie- } \\
\text { ría, Arqui- } \\
\text { tectura, } \\
\text { Urba- } \\
\text { nismo y } \\
\text { afines }\end{array}$ & 42 & 7 & 1 & 4 & 16 & 6 & 8 & 7 & 0 & 2 & 67 & 26 \\
\hline $\begin{array}{l}\text { Matemá- } \\
\text { ticas y } \\
\text { ciencias } \\
\text { Naturales }\end{array}$ & 0 & 1 & 0 & 2 & 1 & 0 & 1 & 1 & 0 & 0 & 2 & 4 \\
\hline & 131 & 30 & 23 & 11 & 144 & 133 & 42 & 121 & 1 & 6 & 341 & 301 \\
\hline
\end{tabular}

Fuente: SNIES, El área académica que predomina en programas de educación a distancia, es Ciencias de la educación, seguida de Economía, Administración, Contaduría y afines e Ingeniería. 
Olga Lilihet M atallana Kuan y M arleny Torres Zamudio Caracterización de la educación superior a distancia, en las universidades colombianas, artículo producto de la investigación

Las universidades oficicial es cuentan con un mayor número de programas en lasáreas de Economía, A dministración, Contaduría y afines, y Ciencias de la educacion, y con un menor numero de programas en oferta para las areas de Matematicas y ciencias naturales y Bellas Artes. L as universidades privadas central izan su oferta en las areas de Ciencias de la educacion y Economia, A dministracion, Contaduria y afines (Ver tabla 3).

El área académica que predomina en programas de educación a distancia es Ciencias de la educación, seguida de Economía, Administracion, Contaduria y afines e Ingeniería, A rquitectura, Urbanismo y afines. En el nivel de formación tecnológica y técnica profesional, el area de Economia, Contaduria y afines es la mas representativa. En el nivel de estudios universitarios, el mavor numero de programas se ubica en el área de la educacion., Las maestrias que cuentan con muy pocos programas en el pais estan distribuidas en, las areas de Educacion, Economia, Administración, Contaduria y afines e ingenieria, Arquitectura, Urbanismo y afines y Ciencias de la salud.

e) Número de programas activos e inactivos por área académica

Los programas activos cuentan con estudiantes matriculados; los inactivos son programas que por reglamentacion del Ministerio de Educacion Nacional no pueden recibir al umnos nuevos y deben garantizar la cul minacion de los programas a los estudiantes que tengan mátriculados. Cabe aclarar que en los datos tomados por área académica no aparecen 34 programas de los 676 registrados. En el nivel de formacion técnica profesional, todos los programas se encuentran activos: a nivel de tecnologla, hay, 121 programas activos; a nivel universitario, 194 programas;' en especialización, 123 programas y en maestría todos los programas estan activos. L as areas académicas con mayor numero de programas inactivos son: Economía Administracion, Contaduria y afines e Ingenieria, A rquitectura, Urbanismo y afines (Ver tabla 4).

Tabla 4. Programas activos e inactivos

\begin{tabular}{|c|c|c|c|c|c|c|c|c|c|c|c|c|}
\hline \multirow{3}{*}{$\begin{array}{l}\text { Año } 2009 \\
\text { Área }\end{array}$} & \multicolumn{6}{|c|}{ Pregrado } & \multicolumn{4}{|c|}{ Postgrado } & \multirow{2}{*}{\multicolumn{2}{|c|}{ Total }} \\
\hline & \multicolumn{2}{|c|}{ Tecnológica } & \multicolumn{2}{|c|}{$\begin{array}{l}\text { Técnica } \\
\text { profesional }\end{array}$} & \multicolumn{2}{|c|}{ Universitaria } & \multicolumn{2}{|c|}{$\begin{array}{l}\text { Especializa- } \\
\text { ción }\end{array}$} & \multicolumn{2}{|c|}{ Maestría } & & \\
\hline & Activo & $\begin{array}{l}\text { Inacti- } \\
\text { vo }\end{array}$ & Activo & $\begin{array}{l}\text { Inac- } \\
\text { tivo }\end{array}$ & $\begin{array}{l}\text { Acti- } \\
\text { vo }\end{array}$ & $\begin{array}{l}\text { Inacti- } \\
\text { vo }\end{array}$ & $\begin{array}{l}\text { Acti- } \\
\text { vo }\end{array}$ & $\begin{array}{l}\text { Inac- } \\
\text { tivo }\end{array}$ & $\begin{array}{l}\text { Acti- } \\
\text { vo }\end{array}$ & $\begin{array}{l}\text { Inacti- } \\
\text { vo }\end{array}$ & $\begin{array}{l}\text { Acti- } \\
\text { vo }\end{array}$ & $\begin{array}{l}\text { Inac- } \\
\text { tivo }\end{array}$ \\
\hline $\begin{array}{l}\text { Agro- } \\
\text { nomía, } \\
\text { veterinaria } \\
\text { y afines }\end{array}$ & 20 & 9 & 4 & 0 & 2 & 2 & 6 & 0 & 0 & 0 & 32 & 11 \\
\hline
\end{tabular}




\section{Revista de}

investigaciones UNAD

Volumen 10. Número 1. Junio 2011

\begin{tabular}{|c|c|c|c|c|c|c|c|c|c|c|c|c|}
\hline \multirow{3}{*}{$\begin{array}{l}\text { Año } 2009 \\
\text { Área }\end{array}$} & \multicolumn{6}{|c|}{ Pregrado } & \multicolumn{4}{|c|}{ Postgrado } & \multirow{2}{*}{\multicolumn{2}{|c|}{ Total }} \\
\hline & \multicolumn{2}{|c|}{ Tecnológica } & \multicolumn{2}{|c|}{$\begin{array}{c}\text { Técnica } \\
\text { profesional }\end{array}$} & \multicolumn{2}{|c|}{ Universitaria } & \multicolumn{2}{|c|}{$\begin{array}{c}\text { Especializa- } \\
\text { ción }\end{array}$} & \multicolumn{2}{|c|}{ Maestría } & & \\
\hline & Activo & $\begin{array}{l}\text { Inacti- } \\
\text { vo }\end{array}$ & Activo & $\begin{array}{l}\text { Inac- } \\
\text { tivo }\end{array}$ & $\begin{array}{l}\text { Acti- } \\
\text { vo }\end{array}$ & $\begin{array}{l}\text { Inacti- } \\
\text { vo }\end{array}$ & $\begin{array}{l}\text { Acti- } \\
\text { vo }\end{array}$ & $\begin{array}{l}\text { Inac- } \\
\text { tivo }\end{array}$ & $\begin{array}{l}\text { Acti- } \\
\text { vo }\end{array}$ & $\begin{array}{l}\text { Inacti- } \\
\text { vo }\end{array}$ & $\begin{array}{l}\text { Acti- } \\
\text { vo }\end{array}$ & $\begin{array}{l}\text { Inac- } \\
\text { tivo }\end{array}$ \\
\hline $\begin{array}{l}\text { Bellas } \\
\text { artes }\end{array}$ & 0 & 1 & 0 & 0 & 1 & 0 & 0 & 0 & 0 & 0 & 1 & 1 \\
\hline $\begin{array}{l}\text { Ciencias } \\
\text { de la Edu- } \\
\text { cación }\end{array}$ & 0 & 0 & 0 & 0 & 145 & 10 & 59 & 11 & 2 & 0 & 206 & 21 \\
\hline $\begin{array}{l}\text { Ciencias } \\
\text { de la } \\
\text { Salud }\end{array}$ & 9 & 1 & 0 & 0 & 8 & 1 & 1 & 1 & 1 & 0 & 19 & 3 \\
\hline $\begin{array}{l}\text { Ciencias } \\
\text { sociales y } \\
\text { humanas }\end{array}$ & 4 & 1 & 0 & 0 & 11 & 2 & 9 & 3 & 0 & 0 & 24 & 6 \\
\hline $\begin{array}{l}\text { Eco- } \\
\text { nomía, } \\
\text { Adminis- } \\
\text { tración, } \\
\text { conta- } \\
\text { duría y } \\
\text { afines }\end{array}$ & 58 & 8 & 23 & 0 & 53 & 19 & 40 & 7 & 2 & 0 & 185 & 34 \\
\hline $\begin{array}{l}\text { Ingeniería, } \\
\text { Arquitec- } \\
\text { tura, } \\
\text { Urba- } \\
\text { nismo y } \\
\text { afines }\end{array}$ & 34 & 15 & 5 & 0 & 19 & 3 & 6 & 9 & 2 & 0 & 66 & 27 \\
\hline $\begin{array}{l}\text { Matemá- } \\
\text { ticas y } \\
\text { ciencias } \\
\text { Naturales }\end{array}$ & 1 & 0 & 2 & 0 & 1 & 0 & 1 & 1 & 0 & 0 & 5 & 1 \\
\hline $\begin{array}{l}\text { No regis- } \\
\text { trados }\end{array}$ & & & & & & & & & & & & \\
\hline
\end{tabular}




\section{Olga Lilihet M atallana Kuan y M arleny Torres Zamudio}

\begin{tabular}{|c|c|c|c|c|c|c|c|c|c|c|c|c|}
\hline \multirow{3}{*}{$\begin{array}{l}\text { Año } 2009 \\
\text { Área }\end{array}$} & \multicolumn{6}{|c|}{ Pregrado } & \multicolumn{4}{|c|}{ Postgrado } & \multirow{2}{*}{\multicolumn{2}{|c|}{ Total }} \\
\hline & \multicolumn{2}{|c|}{ Tecnológica } & \multicolumn{2}{|c|}{$\begin{array}{c}\text { Técnica } \\
\text { profesional }\end{array}$} & \multicolumn{2}{|c|}{ Universitaria } & \multicolumn{2}{|c|}{$\begin{array}{l}\text { Especializa- } \\
\text { ción }\end{array}$} & \multicolumn{2}{|c|}{ Maestría } & & \\
\hline & Activo & $\begin{array}{l}\text { Inac- } \\
\text { tivo }\end{array}$ & Activo & $\begin{array}{l}\text { Inac- } \\
\text { tivo }\end{array}$ & Activo & Inactivo & $\begin{array}{l}\text { Acti- } \\
\text { vo }\end{array}$ & Inactivo & $\begin{array}{l}\text { Acti- } \\
\text { vo }\end{array}$ & Inactivo & Activo & $\begin{array}{l}\text { Inac- } \\
\text { tivo }\end{array}$ \\
\hline Total & 126 & 35 & 34 & 0 & 240 & 37 & 131 & 32 & 7 & 0 & 538 & 104 \\
\hline
\end{tabular}

Fuente: SNIES, los programas activos hacen referencia a programas con estudiantes matriculados. Los inactivos, a programas que por reglamentación del Ministerio de Educación Nacional no pueden recibir alumnos nuevos y deben garantizar su culminación a los estudiantes que tengan matriculados.

\section{f) Programas acreditados por área académica}

Tabla 5. Programas acreditados

\begin{tabular}{|c|c|c|c|c|c|c|c|c|c|c|c|c|c|}
\hline \multirow{3}{*}{$\begin{array}{l}\text { Año } \\
2009 \\
\text { Área }\end{array}$} & \multirow{3}{*}{$\begin{array}{l}\text { Acredita- } \\
\text { ción }\end{array}$} & \multicolumn{6}{|c|}{ Pregrado } & \multicolumn{4}{|c|}{ Postgrado } & \multicolumn{2}{|c|}{ Total } \\
\hline & & \multicolumn{2}{|c|}{$\begin{array}{l}\text { Tecnoló- } \\
\text { gica }\end{array}$} & \multicolumn{2}{|c|}{$\begin{array}{c}\text { Técnica } \\
\text { profesional }\end{array}$} & \multicolumn{2}{|c|}{$\begin{array}{c}\text { Universi- } \\
\text { taria }\end{array}$} & \multicolumn{2}{|c|}{$\begin{array}{c}\text { Especializa- } \\
\text { ción }\end{array}$} & \multicolumn{2}{|c|}{ Maestría } & & \\
\hline & & Oficial & $\begin{array}{l}\text { Pri- } \\
\text { vada }\end{array}$ & Oficial & $\begin{array}{l}\text { Pri- } \\
\text { vada }\end{array}$ & Oficial & $\begin{array}{l}\text { Pri- } \\
\text { vada }\end{array}$ & Oficial & $\begin{array}{c}\text { Priva- } \\
\text { da }\end{array}$ & Oficial & $\begin{array}{l}\text { Priva- } \\
\text { da }\end{array}$ & & \\
\hline \multirow{2}{*}{$\begin{array}{c}\text { Agro- } \\
\text { nomía, } \\
\text { veterina- } \\
\text { ria y }\end{array}$} & $\begin{array}{l}\text { Registro } \\
\text { calificado }\end{array}$ & 16 & 4 & 4 & 0 & 2 & 0 & 1 & 4 & 0 & 0 & 23 & 8 \\
\hline & $N / A$ & 9 & 0 & 0 & 0 & 2 & 0 & 1 & 0 & 0 & 0 & 12 & 0 \\
\hline \multirow[t]{2}{*}{$\begin{array}{l}\text { Bellas } \\
\text { artes }\end{array}$} & $\begin{array}{l}\text { Registro } \\
\text { calificado }\end{array}$ & 0 & 0 & 0 & 0 & 59 & 66 & 10 & 26 & 0 & 2 & 69 & 94 \\
\hline & $\mathrm{N} / \mathrm{A}$ & 0 & 0 & 0 & 0 & 12 & 18 & 2 & 32 & 0 & 0 & 14 & 50 \\
\hline \multirow{2}{*}{$\begin{array}{c}\text { Ciencias } \\
\text { de la } \\
\text { Salud }\end{array}$} & $\begin{array}{l}\text { Registro } \\
\text { calificado }\end{array}$ & 5 & 2 & 0 & 0 & 1 & 0 & 1 & 0 & 1 & 0 & 8 & 2 \\
\hline & $\mathrm{N} / \mathrm{A}$ & 3 & 0 & 0 & 0 & 8 & 0 & 1 & 0 & 0 & 0 & 12 & 0 \\
\hline
\end{tabular}


Revista de

investigaciones UNAD

Volumen 10. Número 1. Junio 2011

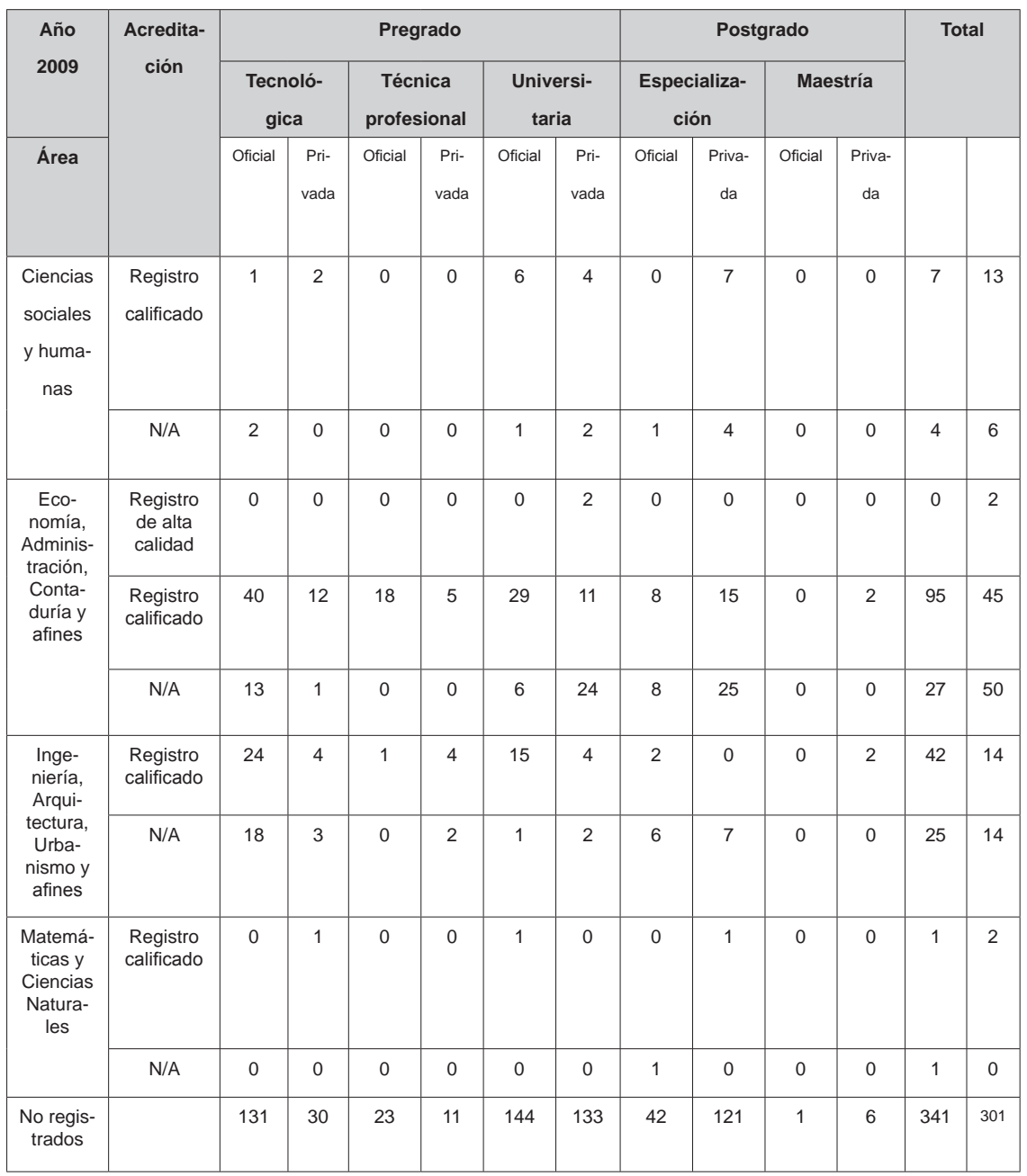

Fuente: SNIES El mayor número de programas, N/A, está en las áreas de Economía, Administración, Contaduría y afines y en Ciencias de la educación.

En educación a distancia, los únicos programas acreditados con registro de alta calidad, ofrecidos por universidades con metodologia a distancia, son universitarios y corresponden a los programas de Administración de empresas en la Ean y Contaduria Pública en la Universidad Militar Nueva Granada, De los 676 programas a distancia que existen, 426 cuentan con registro calificcado, acreditando las condiciones minimas de calidad para el adecuado funcionamiento y 216 figuran como no acreditados. El mayor numero de programas, N/A, está en las áreas de Economía, A dministracion, Contaduría y afines y Ciencias de la educación (Ver tabla 5). 
Olga Lilihet M atallana Kuan y M arleny Torres Zamudio

Caracterización de la educación superior a distancia, en las universidades colombianas, artículo

producto de la investigación

Estudiantes matriculados

a) Número de estudiantes matriculados en metodología a distancia en el periodo 2001-2008

Tabla 6. Matrículas

\begin{tabular}{|c|c|c|c|c|c|c|c|c|}
\hline Año & $\mathbf{2 . 0 0 1}$ & $\mathbf{2 . 0 0 2}$ & $\mathbf{2 . 0 0 3}$ & $\mathbf{2 . 0 0 4}$ & $\mathbf{2 . 0 0 5}$ & $\mathbf{2 . 0 0 6}$ & $\mathbf{2 . 0 0 7}$ & $\mathbf{2 . 0 0 8}$ \\
\hline $\begin{array}{c}\text { NO PRE- } \\
\text { SENCIAL (A } \\
\text { DISTANCIA) }\end{array}$ & 97.961 & 84.635 & 85.927 & 92.627 & 103.682 & 100.487 & 127.883 & 148.230 \\
\hline $\begin{array}{c}\text { SIN } \\
\text { CLASIFICAR }\end{array}$ & 0 & 0 & 0 & 0 & 0 & 0 & 1,257 & 1,279 \\
\hline $\begin{array}{c}\text { TOTAL } \\
\text { NACIONAL }\end{array}$ & 977.243 & 896.823 & 1.050 .032 & 1.113 .726 & 1.195 .724 & 1.282 .934 & 1.363 .129 & 1.483 .631 \\
\hline $\begin{array}{c}\text { PORCENTAJE } \\
\text { DE REPRE- } \\
\text { SENTACIÓN }\end{array}$ & 10,0 & 9,4 & 8,2 & 8,3 & 8,7 & 7,8 & 9,4 & 10,0 \\
\hline
\end{tabular}

Fuente: SNIES, el número de estudiantes que escogen la modalidad a distancia ha pasado de 977.243 en 2001 a 1.483 .631 en 2008

En lo que se refiere a cobertura, la educación a distancia representa actual mente un $10 \%$ del total de estudiantes matriculados en el pais. Sin embargo, es bueno clarificar que en el periodo analizado ha habido un incremento sustancial en cuanto a matrículas de educacion superior, y aunque se mantiene el porcentaje, el numero de estudiantes que escogen esta modalidad educativa casi se ha duplicado desde 2001 (Ver tabla 6).

Graduados

a) Graduados a distancia respecto a graduados a nivel nacional, año 2008

Tabla 7. Graduados

\begin{tabular}{|c|c|c|c|c|}
\hline $\begin{array}{c}\text { NIVEL DE } \\
\text { FORMACIÓN }\end{array}$ & $\begin{array}{c}\text { GRADUADOS A } \\
\text { DISTANCIA }\end{array}$ & $\begin{array}{c}\text { \% GRADUADOS } \\
\text { A DISTANCIA }\end{array}$ & $\begin{array}{c}\text { TOTAL } \\
\text { GRADUADOS }\end{array}$ & \% GRADUADOS \\
\hline $\begin{array}{c}\text { FORMAC. TÉCNICA } \\
\text { PROFESIONAL }\end{array}$ & 405 & 0,25 & 14.076 & 8,64 \\
\hline
\end{tabular}


Revista de

investigaciones UNAD

Volumen 10. Número 1. Junio 2011

\begin{tabular}{|c|c|c|c|c|}
\hline $\begin{array}{c}\text { NIVEL DE } \\
\text { FORMACIÓN }\end{array}$ & $\begin{array}{c}\text { GRADUADOS A } \\
\text { DISTANCIA }\end{array}$ & $\begin{array}{c}\text { \% GRADUADOS } \\
\text { A DISTANCIA }\end{array}$ & $\begin{array}{c}\text { TOTAL } \\
\text { GRADUADOS }\end{array}$ & \% GRADUADOS \\
\hline TECNOLÓGICA & 3.182 & 1,95 & 17.360 & 10,66 \\
\hline UNIVERSITARIA & 8.013 & 4,92 & 96.450 & 59,22 \\
\hline ESPECIALIZACIÓN & 7.124 & 4,37 & 31.973 & 19,63 \\
\hline MAESTRÍA & 40 & 0,02 & 2.911 & 1,79 \\
\hline DOCTORADO & 0 & 0,00 & 98 & 0,06 \\
\hline TOTAL & 18.764 & 11,52 & 162.868 & 100,00 \\
\hline
\end{tabular}

Fuente: Observatorio laboral para la educación, año 2008. El porcentaje más alto de graduados se dio en los niveles de formación universitaria y de especialización.

La tabla 7 indica que de 162.868 estudiantes graduados en el país en 2008, solamente, un $11.52 \%$ correspondio a estudiantes graduados segun la metodología a distancia. El porcentaje más alto de graduados a distancia se dio en los niveles de formacion universitaria y de especializacion.

b) Graduados a distancia por área académica, año 2008

Tabla 8. Graduados por áreas

\begin{tabular}{|c|c|c|c|c|c|c|c|c|c|}
\hline AREA & 2001 & 2002 & 2003 & 2004 & 2005 & 2006 & 2007 & 2008 & Total \\
\hline $\begin{array}{l}\text { AGRONOMÍA } \\
\text { VETERINARIA Y } \\
\text { AFINES }\end{array}$ & 314,00 & 421,00 & 471,00 & 398,00 & 413,00 & 413,00 & 585,00 & 500,00 & $3.515,00$ \\
\hline BELLAS ARTES & 0,00 & 0,00 & 7,00 & 36,00 & 51,00 & 47,00 & 72,00 & 120,00 & 333,00 \\
\hline $\begin{array}{l}\text { CIENCIAS DE LA } \\
\text { EDUCACIÓN }\end{array}$ & $11.853,00$ & $7.826,00$ & $8.375,00$ & $5.701,00$ & $2.263,00$ & $1.890,00$ & $3.420,00$ & $4.236,00$ & $45.564,00$ \\
\hline $\begin{array}{c}\text { CIENCIAS DE LA } \\
\text { SALUD }\end{array}$ & 586,00 & 275,00 & 552,00 & 275,00 & 293,00 & 224,00 & 249,00 & 281,00 & $2.735,00$ \\
\hline $\begin{array}{l}\text { CIENCIAS SOCIA- } \\
\text { LES Y HUMANAS }\end{array}$ & 952,00 & 755,00 & $1.151,00$ & 680,00 & $1.521,00$ & $2.350,00$ & $3.940,00$ & $3.138,00$ & $14.487,00$ \\
\hline $\begin{array}{c}\text { ECONOMÍA, AD- } \\
\text { MINISTRACIÓN, } \\
\text { CONTADURÍAY } \\
\text { AFINES }\end{array}$ & $5.705,00$ & $4.871,00$ & $5.971,00$ & $6.203,00$ & $6.338,00$ & $5.871,00$ & $8.362,00$ & $6.121,00$ & $49.442,00$ \\
\hline $\begin{array}{l}\text { INGENIERÍA, } \\
\text { ARQUITECTURA, } \\
\text { URBANISMO Y } \\
\text { AFINES }\end{array}$ & $2.237,00$ & $1.791,00$ & $1.148,00$ & $1.455,00$ & $1.837,00$ & $2.099,00$ & $3.142,00$ & $3.972,00$ & $17.681,00$ \\
\hline
\end{tabular}


Olga Lilihet Matallana Kuan y M arleny Torres Zamudio

Caracterización de la educación superior a distancia, en las universidades colombianas, artículo producto de la investigación

\begin{tabular}{|c|c|c|c|c|c|c|c|c|c|}
\hline AREA & $\mathbf{2 0 0 1}$ & $\mathbf{2 0 0 2}$ & $\mathbf{2 0 0 3}$ & $\mathbf{2 0 0 4}$ & $\mathbf{2 0 0 5}$ & $\mathbf{2 0 0 6}$ & $\mathbf{2 0 0 7}$ & $\mathbf{2 0 0 8}$ & Total \\
\hline $\begin{array}{c}\text { MATEMÁTICAS } \\
\text { Y CIENCIAS } \\
\text { NATURALES }\end{array}$ & 11,00 & 5,00 & 18,00 & 7,00 & 9,00 & 9,00 & 16,00 & 1,00 & 76,00 \\
\hline SIN CLASIFICAR & 0,00 & 0,00 & 0,00 & 132,00 & 185,00 & 195,00 & 332,00 & 395,00 & $1.239,00$ \\
\hline Total & $21.658,00$ & $15.944,00$ & $17.693,00$ & $14.887,00$ & $12.910,00$ & $13.098,00$ & $20.118,00$ & $18.764,00$ & $135.072,00$ \\
\hline
\end{tabular}

Fuente: Observatorio laboral para la educación, año 2008. En el periodo analizado hay un mayor número de graduados en el área de Economía, Administración, Contaduría y afines.

En el período analizado 2001-2008, el mavor número de estudiantes se ha graduado en las áreas de Economia, A dministracion Contaduria y afines, sequido de ciencias de la educacion. Al analizar el comportamiento anual desde 2001 hasta 2003, primaban los grados en Ciencias de la educacion pero desde 2004 esta tendencia, se revierte y se posicionan las áreas de Economia, Administracion Contaduria y afines.

c) G raduados hasta 2008 con metodología a distancia en pregrado y postgrado por departamento.

Tabla 9. Graduados por departamento

\begin{tabular}{|c|c|c|c|c|c|c|c|c|c|}
\hline \multirow{2}{*}{$\begin{array}{l}\text { ZONA } \\
\text { GEO- } \\
\text { GRÁfl- } \\
\text { CA }\end{array}$} & \multicolumn{8}{|c|}{ PERIODO DE GRADUACION } & \multirow[t]{2}{*}{ TOTAL } \\
\hline & 2001 & 2002 & 2003 & 2004 & 2005 & 2006 & 2007 & 2008 & \\
\hline $\begin{array}{l}\text { ANTIO- } \\
\text { QUIA }\end{array}$ & 564,00 & 532,00 & 754,00 & 732,00 & 892,00 & $1.175,00$ & 759,00 & $1.038,00$ & $6.446,00$ \\
\hline $\begin{array}{l}\text { ATLÁN- } \\
\text { TICO }\end{array}$ & 113,00 & 112,00 & 84,00 & 61,00 & 27,00 & 1,00 & 0,00 & 6,00 & 404,00 \\
\hline $\begin{array}{l}\text { BOGO- } \\
\text { TÁ, } \\
\text { D. C. }\end{array}$ & $9.855,00$ & $5.822,00$ & $5.859,00$ & $4.849,00$ & $6.846,00$ & $7.434,00$ & $11.734,00$ & $10.126,00$ & $62.525,00$ \\
\hline $\begin{array}{l}\text { BOLÍ- } \\
\text { VAR }\end{array}$ & 207,00 & 289,00 & 439,00 & 481,00 & 278,00 & 171,00 & 656,00 & 247,00 & $2.768,00$ \\
\hline BOYACÁ & $1.230,00$ & $1.097,00$ & $2.497,00$ & $1.076,00$ & 574,00 & 547,00 & 637,00 & 770,00 & $8.428,00$ \\
\hline CALDAS & $2.461,00$ & $2.254,00$ & 718,00 & 443,00 & 212,00 & 513,00 & $1.623,00$ & $2.050,00$ & $10.274,00$ \\
\hline $\begin{array}{l}\text { CAQUE- } \\
\text { TÁ }\end{array}$ & 0,00 & 0,00 & 0,00 & 0,00 & 0,00 & 19,00 & 11,00 & 30,00 & 60,00 \\
\hline CAUCA & 0,00 & 0,00 & 0,00 & 0,00 & 7,00 & 15,00 & 25,00 & 24,00 & 71,00 \\
\hline CHOCÓ & 152,00 & 31,00 & 255,00 & 86,00 & 0,00 & 9,00 & 42,00 & 0,00 & 575,00 \\
\hline $\begin{array}{l}\text { CÓRDO- } \\
\text { BA }\end{array}$ & $1.038,00$ & 77,00 & 15,00 & 806,00 & 0,00 & 21,00 & 13,00 & 14,00 & $1.984,00$ \\
\hline
\end{tabular}


Revista de

investigaciones UNAD

Volumen 10. Número 1. Junio 2011

\begin{tabular}{|c|c|c|c|c|c|c|c|c|c|}
\hline \multirow{2}{*}{$\begin{array}{l}\text { ZONA } \\
\text { GEO- } \\
\text { GRÁfICA }\end{array}$} & \multicolumn{8}{|c|}{ PERIODO DE GRADUACION } & \multirow[t]{2}{*}{ TOTAL } \\
\hline & 2001 & 2002 & 2003 & 2004 & 2005 & 2006 & 2007 & 2008 & \\
\hline $\begin{array}{l}\text { CUNDI- } \\
\text { NAMAR- } \\
\text { CA }\end{array}$ & 325,00 & 276,00 & 123,00 & 106,00 & 56,00 & 67,00 & 76,00 & 22,00 & $1.051,00$ \\
\hline $\begin{array}{l}\text { GUAJI- } \\
\text { RA }\end{array}$ & 38,00 & 461,00 & 377,00 & 68,00 & 0,00 & 0,00 & 12,00 & 146,00 & $1.102,00$ \\
\hline HUILA & 7,00 & 0,00 & 0,00 & 42,00 & 0,00 & 0,00 & 0,00 & 0,00 & 49,00 \\
\hline $\begin{array}{l}\text { MAGDA- } \\
\text { LENA }\end{array}$ & 361,00 & 110,00 & 130,00 & 69,00 & 14,00 & 3,00 & 73,00 & 71,00 & 831,00 \\
\hline NARIÑO & 0,00 & 4,00 & 3,00 & 5,00 & 44,00 & 133,00 & 207,00 & 384,00 & 780,00 \\
\hline $\begin{array}{l}\text { NORTE } \\
\text { DE SAN- } \\
\text { TANDER }\end{array}$ & 502,00 & 619,00 & $1.661,00$ & $1.701,00$ & 533,00 & 579,00 & $1.198,00$ & $1.545,00$ & $8.338,00$ \\
\hline $\begin{array}{l}\text { QUIN- } \\
\text { DíO }\end{array}$ & 339,00 & 372,00 & 418,00 & 508,00 & 229,00 & 122,00 & 232,00 & 225,00 & $2.445,00$ \\
\hline $\begin{array}{l}\text { RISA- } \\
\text { RALDA }\end{array}$ & 94,00 & 293,00 & 619,00 & 122,00 & 0,00 & 16,00 & 13,00 & 14,00 & $1.171,00$ \\
\hline $\begin{array}{l}\text { SAN- } \\
\text { TANDER }\end{array}$ & 919,00 & 732,00 & 978,00 & 770,00 & 614,00 & 199,00 & 968,00 & 465,00 & $5.645,00$ \\
\hline SUCRE & 16,00 & 13,00 & 63,00 & 19,00 & 29,00 & 210,00 & 95,00 & 171,00 & 616,00 \\
\hline TOLIMA & $2.115,00$ & $1.867,00$ & $2.135,00$ & $2.259,00$ & $2.159,00$ & $1.603,00$ & $1.591,00$ & $1.290,00$ & $15.019,00$ \\
\hline $\begin{array}{l}\text { VALLE } \\
\text { DEL } \\
\text { CAUCA }\end{array}$ & $1.322,00$ & 983,00 & 565,00 & 684,00 & 396,00 & 261,00 & 153,00 & 126,00 & $4.490,00$ \\
\hline Total & $21.658,00$ & $15.944,00$ & $17.693,00$ & $14.887,00$ & $12.910,00$ & $13.098,00$ & $20.118,00$ & $18.764,00$ & $135.072,00$ \\
\hline
\end{tabular}

Fuente: Observatorio laboral para la educación, año 2008. Bogotá cuenta con el mayor número de estudiantes graduados.

Los estudiantes graduados durante 2008, con la metodología a distancia, se ubican en su mayoria en Bogota y en los departamentos de Caldas, Norte de Santander, Tolima y Antiogula para el periodo 2001-2008. Bogota cuenta con el mayor número de estudiantes graduados;' los departamentos que más han graduado estudiantes segun esta metodologia son Tolima, Caldas y Boyacá.

IBC (ingreso base de cotización al sistema de seguridad social)

a) IBC para formación a Distancia. 
Olga Lilihet M atallana Kuan y M arleny Torres Zamudio

Caracterización de la educación superior a distancia, en las universidades colombianas, artículo

producto de la investigación

Tabla 10. IBC

\begin{tabular}{|c|c|c|c|c|c|}
\hline \multirow{2}{*}{\multicolumn{2}{|c|}{ NIVEL DE ESTUDIO }} & \multicolumn{4}{|c|}{ MEDIDAS } \\
\hline & & \multicolumn{2}{|c|}{$\begin{array}{l}\text { METODOLOGÍA A DISTANCIA } \\
\text { PRESENCIAL }\end{array}$} & \multicolumn{2}{|c|}{ METODOLOGÍA } \\
\hline $\begin{array}{l}\text { NIVEL } \\
\text { ACADÉMICO }\end{array}$ & $\begin{array}{l}\text { NIVEL DE } \\
\text { FORMACIÓN }\end{array}$ & IBC & $\begin{array}{l}\text { TASA DE } \\
\text { COTIZACIÓN }\end{array}$ & IBC & $\begin{array}{l}\text { TASA DE } \\
\text { COTIZACIÓN }\end{array}$ \\
\hline \multirow[t]{4}{*}{ PREGRADO } & PREGRADO & $1.098 .646,00$ & 0,71 & $1.171 .511,00$ & 0,70 \\
\hline & $\begin{array}{l}\text { FORMACIÓN } \\
\text { TÉCNICA } \\
\text { PROFESIONAL }\end{array}$ & $924.269,00$ & 0,61 & $792.498,00$ & 0,66 \\
\hline & TECNOLÓGICA & $992.483,00$ & 0,72 & $929.615,00$ & 0,69 \\
\hline & UNIVERSITARIA & $1.167 .462,00$ & 0,72 & $1.258 .609,00$ & 0,70 \\
\hline \multirow[t]{4}{*}{ POSGRADO } & POSGRADO & $1.571 .740,00$ & 0,86 & $2.534 .949,00$ & 0,91 \\
\hline & $\begin{array}{l}\text { ESPECIALIZA- } \\
\text { CIÓN }\end{array}$ & $1.571 .740,00$ & 0,86 & $2.480 .214,00$ & 0,90 \\
\hline & MAESTRÍA & & & $2.818 .220,00$ & 0,92 \\
\hline & DOCTORADO & & & $4.526 .989,00$ & 0,98 \\
\hline
\end{tabular}

Fuente: Observatorio laboral para la educación, IBC por nivel de formación. Graduación 2008, fecha de corte 2009.

Los datos indican que los recién graduados (2008) en la modalidad a distancia del nivel de formacion tecnico profesional y tecnologico ganan $\$ 131.771$ pesos y $\$ 62,868$ pesos más que sus homologos graduados de manera presencial. Los universitarios que trabaian como empleados dependientes recibieron en promedio \$91.147 pesos menos, respecto a los graduados presenciales. Los de nivel de especializacion recibieron \$908.474 pesos menos que sus homólogos presenciales, siendo esta ulltima la diferencia mas representativa.

La tasa de cotización indica el porcentaje de graduados vinculado al sector formal de la economía. En formacion tecnica profesional ese porcentaje es de $61 \%$, en la formación universitaria, el $72 \%$ y en la de especializaciones, el $86 \%$. Es bueno puntual izar queel observatorio laboral ha venido real izando encuestas a los graduados a partir de 2001, que en 2007 la encuesta estuvo disponible en el sitio Web del Observatorio, donde 25 mil graduados la diligenciaron de manera voluntaria. "Cuatro Instituciones de Educacion Superior concentran el $25 \%$ de los graduados que la diligenciaron. Estas Instituciones son las siguientes: Universidad de los Andes (1.905), Universidad de Caldas (1.851), 
Revista de investigaciones UNAD

Volumen 10. Número 1. Junio 2011

Fundación Universidad de Bogotá Jorge Tadeo L ozano (1.539) y Universidad de San Buenaventura (1.038)".

\section{Conclusiones}

En Colombia la educación superior a distancia ha ganado prestigio en los últimos años, tanto que se evidencia en la actualidad una tendencia creciente por.parte de las universidades a ofrecer este tipo de metodología. El $69.6 \%$ de as instituciones de educacion superior acreditadas como universidades, ofrecen programas a distancia;; la gran mayoría de,estas tambien brindan programas con metodologia presencial. Esta metodologia dual hace presumir que no existe una verdadera especializacion en la metodologia a distancia, y que solamente son programas que surgen como respuesta a la creciente demanda de educación superior en el pals.

La UNAD es la única con dedicación exclusiva a la metodología a distancia, pero universidades como la del Quindio, la de Cartagenna, Antonio Nariño y Santo Tomas cuentan con una amplia y cada vez más creciente oferta a distancia. Es importante el papel de las universidades oficiales regionales en la aplicación de la metodologia a distancia en pregrado y la cada vez mayor participacion de las universidades privadas en postgrados. De todas maneras, a nivel de maestria la oferta es escasa, en un terreno poco explorado, que puede ser un mercado potencial, pues son realmente pocas las instituciones que han incursionado en esta area.

En la metodología a distancia están inexploradas las áreas de Matemáticas, Bellas.Artes y ciencias de la salud, lo que constituye un reto importante para las universidades que pueden incursionar con programas en estas áreas. Para ello requerirán materiales didacticos adecuados, que permitan adaptar adelantos tecnologicos que contemplen el empleo de simulacion y realidad virtual para el aprendizaje, y que logren que el estudiante aprenda participando.

Lasmatrículasenlamodalidad estudiadasehan incrementado considerablemente, y todas las previsiones indican que la educacion a distancia continuara con un desarrollo creciente, Todos los grupos etarios y estratos sociales demandan formacion; se han flexibilizado las condiciones de ingreso y permanencia en los programas academicos, gracias a las posibilidades que ofrecen los nuevos medios de comunicacion, los cuales se han convertido en una importante herramienta de escolarizacion permanente que permiten al estudiante atender simultáneamente roles laborales, académicos y familiares.

8 Observatorio Laboral/investigadores, página Web http://www.graduadoscolombia.edu.co/html/1732/article156192.html. 
Olga Lilihet M atallana Kuan y M arleny Torres Zamudio

La educación a distancia ha permitido que muchas regiones del país cuenten con acceso a educacion superior, destacandose además de Bogota, los departamentos de Cal das y Tolima como los que mas han tenido graduados según esta metodologla en el periodo 2001-2008. Existen regiones tradicionalmente marginadas como Choco y Caqueta, que afortunadamente estan hallando soluciones educativas, aunque para que se de una real cobertura, es necesario que el pals supere las limitaciones tecnologicas que dificultan un acceso equitativo a los programas de educacion superior.

La educación a distancia en el país, a pesar de que es una metodología que lleva varios anos, aun no tiene el nivel de impacto que debería tener, dado que debería ser la alternativa de educacion más incluyente, por su estructura que brinda un ambito de aprendizaje unico para personas adultas que laboran o que por diversas circunstancias no pueden acceder a la educacion presencial. Es ahora, con la utilizacion de los medios virtuales, cuando puede dar un gran paso en la consolidacion de contextos educativos que no solo fortal ezcan los conocimientos teoricos sino que potencialicen en sus educandos las competencias laborales que su medio y el pais necesitan.

\section{Referencias bibliográficas}

Asociación Colombiana de Universidades, Ascun. (2007). Políticas y estrategias para la Educación superior de Colombia 2006-2010 Bogotá: Corcas Editores.

Briceño, R. C., (1991). Colombia. En Philip G. Altbach (Ed.), International Higher Education. An Encyclopedia (pp. 150-157). New York, London: Garland Publishing.

Colombia. M inisterio de Educación Nacional. 1992. Ley 30, Por la cual se organiza el servicio Público de la educación superior. Bogotá.

Colombia. Ministerio de Educación Nacional. 2006. Resolución 2755. Bogotá.

González, G. R. (1992) La experiencia colombiana en materia de evaluación de la educación superior. En Evaluación, Promoción de la Calidad y Financiamiento de la Educación Superior (pp. 55-57). Ciudad de México: Publicaciones Anuies.

Instituto Internacional de la Unesco para la Educación Superior en América Latina y el Caribe (lesalc/Unesco). (2006). Informe sobre la educación superior en América Latina y el Caribe, 2000-2005. La metamorfosis de la educación superior". Caracas: M etrópolis.

Misas, A. G. (2004). La educación superior en Colombia. Análisis y Estrategias para su desarrollo. Bogotá: Universidad Nacional de Colombia. 


\section{Revista de investigaciones UNAD Volumen 10. Número 1. Junio 2011}

Montenegro, M. J. (1994). La Educación Superior en Colombia, Revista Anuies, 23(92), 53-59.

Moreno, C. M. (2001). Un modelo educativo para la innovación del aprendizaje. Guadalajara: Universidad de Guadalajara.

Parra, S. R. y Jaramillo, B. (1985). La Educación Superior en Colombia. Caracas: Cresalc-Unesco.

Unesco. 2005. Hacia las Sociedades del Conocimiento. Informe Mundial. París: J ouve, Mayenne.

Zarur M., X. y Rodríguez, C, J. C. (2006). Evolución, situación actual y perspectivas de la educación superior en Colombia 2005. Caracas: Instituto Internacional para la Educación superior en América latina y el Caribe.

\section{Anexo 1. Formato de observación estructurada}

"Caracterización de la educación superior a distancia en las universidades colombianas"

\begin{tabular}{|c|c|c|}
\hline \multicolumn{3}{|c|}{ DATOS GENERALES } \\
\hline \multirow[t]{6}{*}{ FECHA } & $\begin{array}{l}\text { OBSERVACIÓN } \\
\mathrm{N}^{\circ}\end{array}$ & BASE DE DATOS \\
\hline & & Snies \\
\hline & & Observatorio laboral \\
\hline & & ASCUN \\
\hline & & Otra \\
\hline & & Consulta Instituciones programas \\
\hline \multicolumn{3}{|c|}{ Variable: Universidades } \\
\hline & \multicolumn{2}{|c|}{ Graduados matriculados otros } \\
\hline & \multicolumn{2}{|c|}{ Número de universidades en el pais } \\
\hline & \multicolumn{2}{|c|}{ Número de universidades con programas a distancia } \\
\hline & \multicolumn{2}{|c|}{ Nombres de universidades oficiales con programas a distancia } \\
\hline & \multicolumn{2}{|c|}{ Nombres de universidades privadas con programas a distancia } \\
\hline \multicolumn{3}{|c|}{ Variable: Programas } \\
\hline \multicolumn{3}{|c|}{ Número de programas ofrecidos a nivel nacional } \\
\hline \multicolumn{3}{|c|}{ Número de programas ofrecidos en metodologia a distancia } \\
\hline \multicolumn{3}{|c|}{ Número de programas ofrecidos por origen institucional (oficial) } \\
\hline \multicolumn{3}{|c|}{ Número de programas ofrecidos por origen institucional (privado) } \\
\hline \multicolumn{3}{|c|}{ Número de programas ofrecidos por nivel de formación } \\
\hline \multicolumn{3}{|c|}{ Número de programas ofrecidos por universidades oficiales por nivel de formación } \\
\hline \multicolumn{3}{|c|}{ Número de programas oftecidos por universidades privadas por nivel de formación } \\
\hline \multicolumn{3}{|c|}{ Número de programas ofrecidos por área académica, origen y nivel de formación } \\
\hline \multicolumn{3}{|c|}{$\begin{array}{l}\text { Número de programas ofrecidos por área académica y nivel de formación, activos e } \\
\text { inactivos }\end{array}$} \\
\hline \multirow{2}{*}{\multicolumn{3}{|c|}{$\begin{array}{l}\text { Número de programas ofrecidos por área académica y nivel de formación, acreditados y } \\
\text { N/A } \\
\text { Variable: Estudiantes matriculados }\end{array}$}} \\
\hline & & \\
\hline \multicolumn{3}{|c|}{ Estudiantes matriculados en el periodo 2001 a 2008} \\
\hline
\end{tabular}


Olga Lilihet M atallana Kuan y M arleny Torres Zamudio

Caracterización de la educación superior a distancia, en las universidades colombianas, artículo producto de la investigación

\begin{tabular}{|l|l|}
\hline \multicolumn{1}{|l|}{ DATOS GENERALES } \\
\hline FECHA & $\begin{array}{c}\text { OBSERACIÓN } \\
\mathbf{N}^{\circ}\end{array}$ \\
\hline A nivel nacional estudiantes matriculados en el periodo 2001 a 2008 \\
\hline A distancia \\
Variable: graduados \\
\hline Estudiantes graduados en 2008 \\
\hline A nivel nacional estudiantes graduados en 2008, \\
\hline A distancia estudiantes graduados de 2001-2008, por área \\
\hline Estudiantes graduados de 2001-2008, por zona geográfica \\
\hline Variable: IBC \\
\hline IBC año 2008 \\
\hline por nivel de formación \\
\hline Metodologia a distancia \\
\hline IBC año 2008 \\
\hline Por nivel de formación \\
\hline metodologia presencial \\
\hline
\end{tabular}

